\title{
The profile of cyclists in the city of Juiz de Fora
}

\author{
Ugo Castañon ${ }^{\mathrm{a}, *}$, José A. B. Castañon ${ }^{\mathrm{a}}$ and Marcio P. S. Santos ${ }^{\mathrm{b}}$ \\ ${ }^{a}$ Federal University of Juiz de Fora, Engineering Faculty, Room 4156 Campus of UFJF, Juiz de Fora, Minas \\ Gerais, Brasil \\ ${ }^{\mathrm{b}}$ Federal University of Rio de Janeiro, (COPPE/ UFRJ), C.T. - Bloco H Room 106, Rio de Janeiro, Rio de \\ Janeiro, Brasil
}

\begin{abstract}
The problems related to the means of transport in urban Brazilians have been getting worse over the years. The increase of the fleet, to the detriment of the adequacy and extent of the road network of cities contributes to this fact. This paper presents a profile of users of cycling in the city of Juiz de Fora, and thus contributes to the study of sustainable urban mobility, providing researchers with information that can guide the search for the encouragement of the use of means of transport to meet environmental needs urban centers that contributes to social equity.
\end{abstract}

Keywords: bicycles, profile of cyclists, sustainable mobility.

\section{Introduction}

A medium-sized city, with problems of traffic congestion on specific points and times and a predominance of compromising aspects of social equality, such as average income level and removal of peripheral residential neighborhoods, need to consider mobility as an essential factor for democratic access population to all activities offered by the company.

In addition to the serious consequences for the environment by noise pollution and the waste released into the atmosphere by the current use of motorized modes, means the economic burden on the financial cost and the complexity of the planning of urban traffic.

The social aspect also cannot be fully addressed by the cities, since urbanization, in reference to transport, cannot comply fully and promptly the entire geographical environment.

The common use of the bicycle as the main means of transport, contributes significantly to promoting sustainable development, also the mobility of the lower income strata.
The bike is used in several countries, both for its low purchase and maintenance, is the culture of the population, as an option to daily shifts. [5]

A portrait of locomotion in cities can be seen through the information of CNI - National Confederation of Industries. According to the survey, concerning the means of locomotion, $68 \%$ of people use more than one means of transport to make their journeys from home to the place of their activities, and used public transportation as the main means of transport for $42 \%$ of population, the bike reaches $8 \%$ placing it behind the family car that has $16 \%$.[8]

Because of the present non-motorized transport pollutants and noise emissions low, and low risk of accidents to other road users, are often considered key elements of a sustainable transport system. [6]

The need for public policies that encourage nonmotorized means of transport is demonstrated by the fact that not all users who purchase your bike moving through urban traffic because they can sense insecurity.[6]

\footnotetext{
*Corresponding author. E-mail: ugo.castanon@engenharia.ufjf.br
} 
The need for public policies that encourage nonmotorized means of transport is demonstrated by the fact that not all users who purchase your bike moving through urban traffic because they can sense insecurity [6].

The decision to hold a bicycle trip involves not only the characteristics of the trip itself, but the behavior and attitude of the individual in relation to the bicycle (DOT, 1999)

In a study to know the reality of cycling in the city of Cárceres in Mato Grosso, the number found was surprisingly supportive of this non-motorized transport. [4]

By examining the reality of Brazilian cities, it appears that there is already some use of the bicy-

\section{Objective}

Identify the profile of cycling in the city of Juiz de Fora, and brings data to the public policies of urban traffic evaluating the appropriateness of the proposed bicycle use as a motor vehicle, capable of contributing to the preservation of the environment and promote sustainable mobility.

\section{Methodology}

To obtain information about bike users in Juiz de Fora, his behavior in traffic held a field survey during the months March to October 2009. The methodology of Exploratory Survey.

This type of survey has a real purpose, the contribution by collecting data on individuals or their environments, thus allowing obtaining an overview of a particular issue. [2]

The data collection instrument of this Survey, applied to the sample of cyclists was the questionnaire. This was based on the questionnaire used by Yang I Ti, in 1997, on the risk perception of cyclists in relation to the urban traffic system in the city of Rio de Janeiro.

The questionnaire has a division into four distinct stages: Presentation of the questionnaire, Identification of the Bike Rider, Characterization of trips made.

\subsection{The limits of the study area}

The city of Juiz de Fora, the focus of this work shows no initiative to support the movement of bicycles in its road system. A bike path of $2.5 \mathrm{~km}$ cle as a means of transportation to work and study and leisure activities, thus requiring, for appropriate treatment to the role it plays in urban displacement of thousands.

The existence of a characterization of trips by bicycle are included where information on distance, duration and distribution of the same personal characteristics of users, help develop a guide for the planning of spaces and the decision-making, thereby improving the state of practice this area. [7]

The interest of urban managers in expanding the number of trips made by non-motorized vehicle collides with the need to improve the infrastructure for cyclists and pedestrians. [7]

was implanted on the campus of Federal University of Juiz de Fora, when the renovation project of the campus ring road, this being an isolated initiative. This situation reinforces the need for studies like the present work.

\subsection{Sample size}

Although there is no need to ensure the representativeness of the sample in an Exploratory Survey methodology, were used the equation (1) calculate the size of a single sample. [3]

$$
\eta=\left(\frac{Z_{\alpha / 2}}{E}\right)^{2} \cdot 0,25
$$

Therefore, according to equation 1, we obtain the sample size equal to 385 questionnaires. Front of 400 valid questionnaires used, keep the representativeness of the population of cyclists in the city of Juiz de Fora.

\section{Results}

In conducting this Survey were obtained the following results regarding the characterization of the cyclist, there is a predominance of males $(85 \%$ of users). Figure 1 shows a higher concentration of cyclists from 20 to 30 years. In addition, we also show that the younger age groups have a good participation of women, this percentage decreasing with increasing age. 


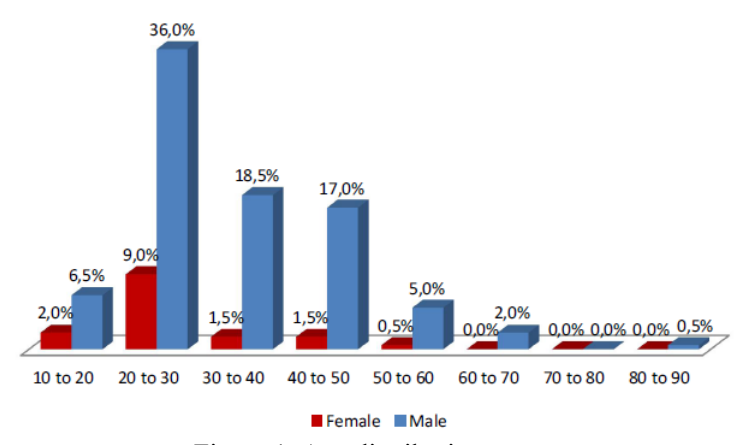

Figure 1. Age distribution

Figure 2 shows the reading of the users, most of them have incomes between R \$ 465.00 and R \$ 2,350.00.

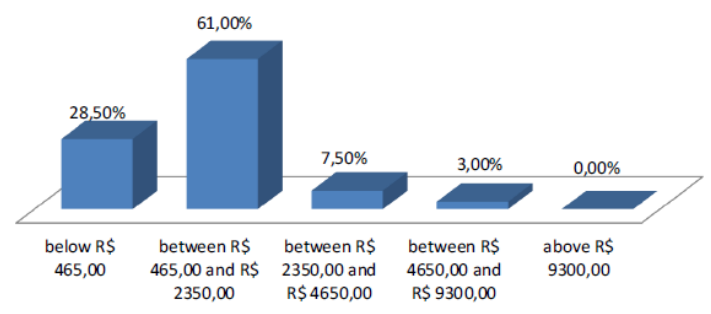

Figure 2. Salary distribution

Figure 3 shows the relationship of time experience in the use of bicycles. The importance of this issue is based on the fact that, despite the difficulties of users of bicycles in a city with a small infrastructure for cyclists, there is this type of user.

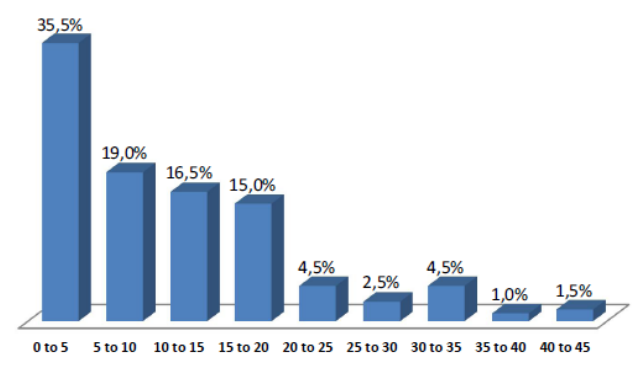

Figure 3. Experience (in years)

As regards the characterization of accidents involving cyclists, was designated a cutting line in 2008. Thus cyclists responded only on accidents that year, not during the entire duration of cycling. Thus, we can observe the results in the Figure 4.

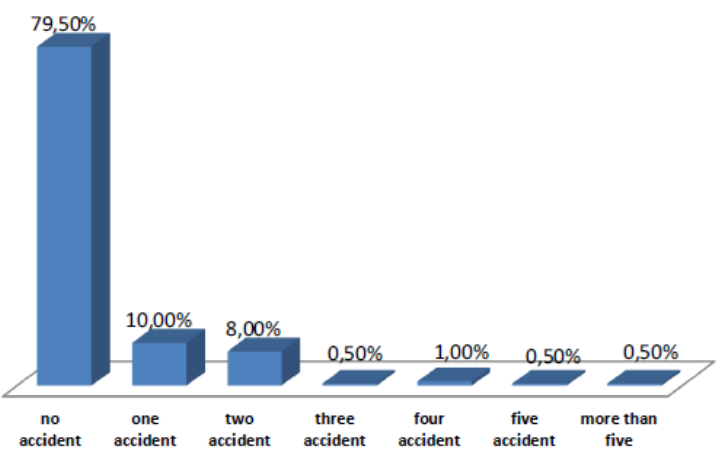

Figure 4. Number of accidents

Can be noted by the data in Figure 5, which is $8.5 \%$ of cyclists involved in accidents with pedestrians. More serious than this, the graph shows the recurrence of such shocks. The location of these shocks was not defined as trampling on the progress on the sidewalk or crosswalks. Therefore, this point has not been identified and requires further studies.

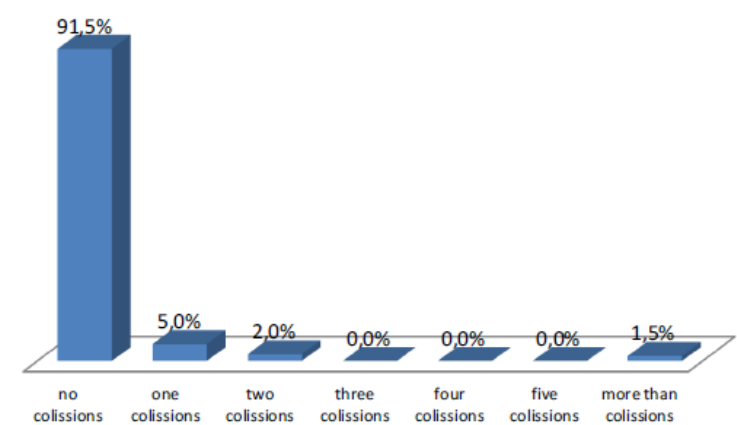

Figure 5. Collisions with pedestrians

Figure 6 show the relation of accidents involving bicycles and cars.

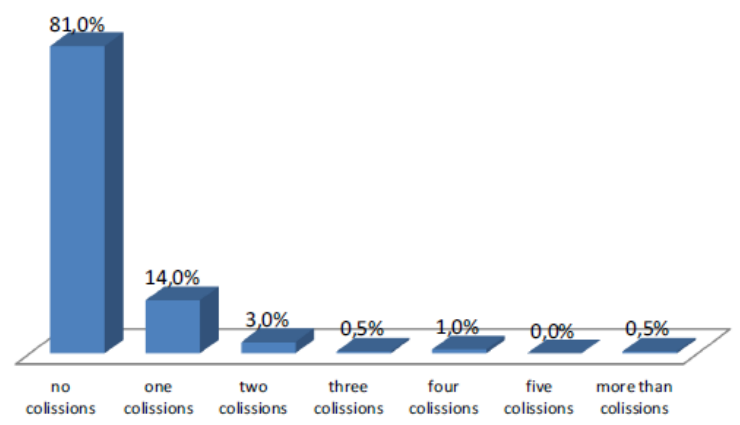

Figure 6. Collisions with cars 
Figure 7 show the relation of accidents involving bicycles and taxis, specifically.

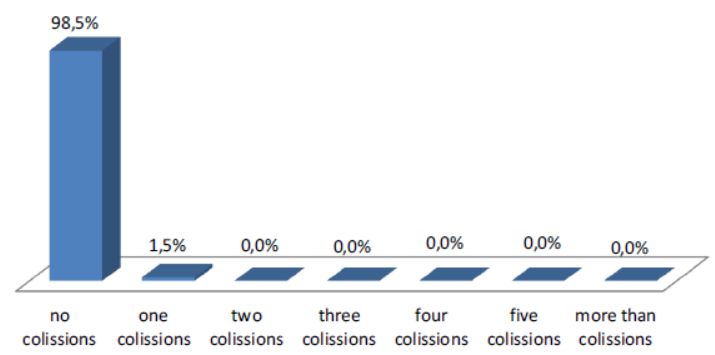

Figure 7. Collisions with taxis

Despite the low rate of accidents involving bicycles and taxis, together with analyzing accidents involving passenger cars and bikes, it is concluded that the share of the small path between vehicles and cyclists generates a large number of accidents $(20.5 \%)$

Asked about the place where the collision between a bicycle and a car parked $57 \%$ of cyclists reported clash with the open doors of the vehicle parked at the curb. Thus, a major cause of accidents involving cars and bicycles should be considered the carelessness of drivers when they open the door, without heed to the movement around it, when parked on the road.

Regarding the characterization and the use of the bicycle as a vehicle of the day to day, and the use of other modes of transport, we sought to further identify the difficulties and limitations that undergoes transport by bicycle in the city of Juiz de Fora, as we see in Figure 8.

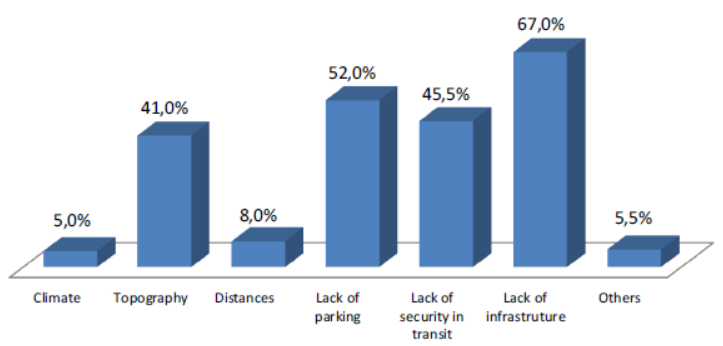

Figure 8. Impedance factors on the use of bicycles

In step characterization of the bicycle, as shown in Figures 9, 10 and 11, we observed the following results regarding the type of bike, mandatory safety equipment and personal safety equipment.

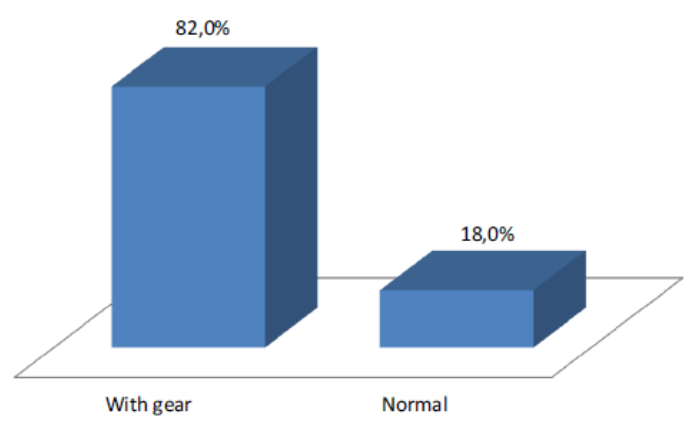

Figure 9. Types of bicycles

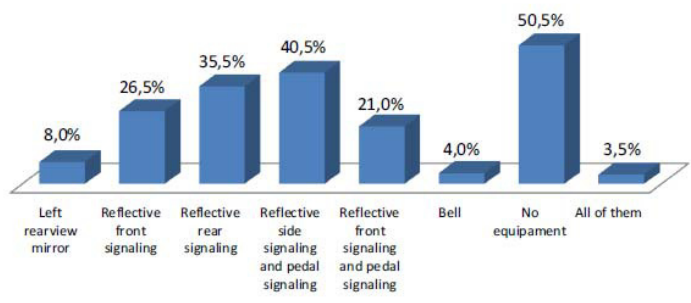

Figure 10. Mandatory safety equipment

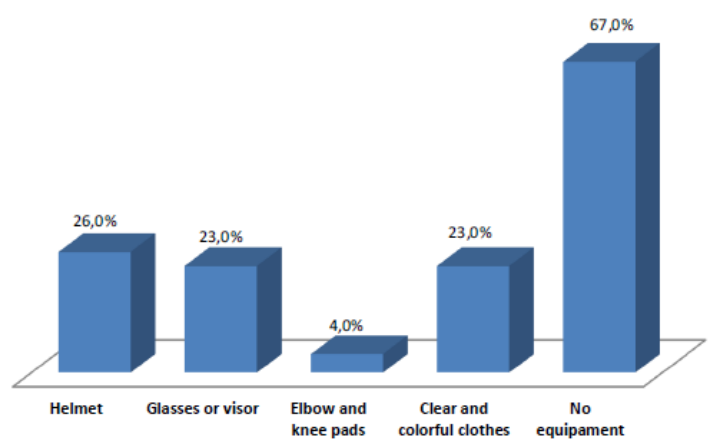

Figure 11. Personal safety equipment

The intersection of these data shows that $33 \%$ use some type of equipment, with the concomitant use of higher frequency is for helmet and appropriate clothing, which is distributed equally in all ages and with greater focus on higher bands salaries. The use of elbow and knee pads is concentrated in younger age ranges between 10 and 20 years.

Step on the characterization of the trips, it was concluded that during the week each rider performs an average of 2.3 trips lasting an average of 29.7 minutes, while on weekends the average number of trips and the time drops to 1.3 running increases to 56.9 minutes. 
Figures 12 and 13 shows the main reasons for each bicycle trip, either during the week or on weekends.

-Work $\square$ School $\square$ Recreation $\square$ Shop -Others

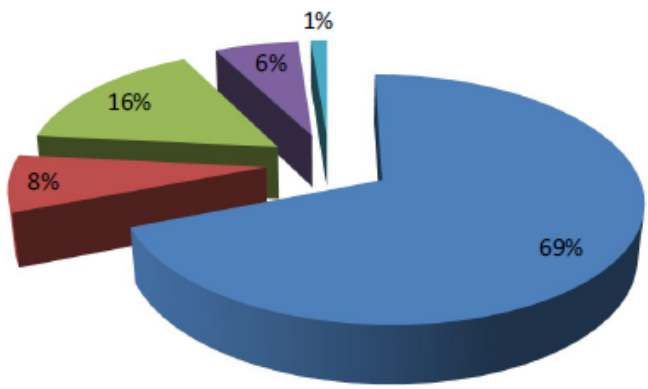

Figure 12. Purposes of travel during the week

Work School Recreation Shop $\square$ Others

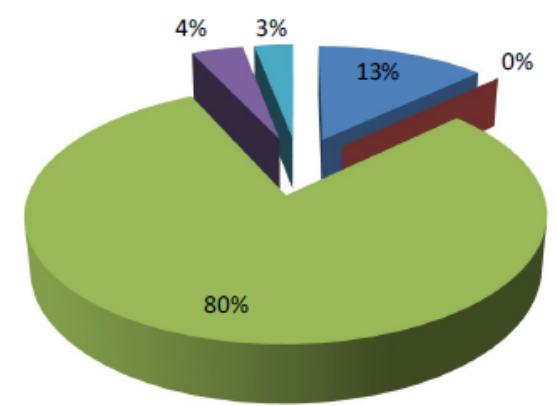

Figure 13. Purposes of travel during the weekends

Table 1 presents the distribution of trips by bicycle, by Administrative Region, the city of Juiz de Fora.

Table 1

$\mathrm{O} / \mathrm{D}$ matrix

\begin{tabular}{|c|c|c|c|c|c|c|c|}
\hline $0 / 0$ & North & South & East & West & Notheast & Southeast & Central \\
\hline North & $3,1 \%$ & $0,9 \%$ & $1,1 \%$ & $1,3 \%$ & $0,2 \%$ & $0,0 \%$ & $2,9 \%$ \\
\hline South & $1,1 \%$ & $0,9 \%$ & $0,9 \%$ & $1,8 \%$ & $0,9 \%$ & $0,7 \%$ & $3,5 \%$ \\
\hline East & $0,9 \%$ & $0,9 \%$ & $2,9 \%$ & $1,5 \%$ & $0,2 \%$ & $0,7 \%$ & $7,5 \%$ \\
\hline West & $1,3 \%$ & $1,8 \%$ & $1,5 \%$ & $6,6 \%$ & $1,3 \%$ & $2,4 \%$ & $3,8 \%$ \\
\hline Notheast & $0,2 \%$ & $0,7 \%$ & $0,2 \%$ & $1,3 \%$ & $2,7 \%$ & $0,2 \%$ & $2,4 \%$ \\
\hline Southeast & $0,0 \%$ & $0,7 \%$ & $0,7 \%$ & $2,4 \%$ & $0,2 \%$ & $0,9 \%$ & $3,5 \%$ \\
\hline Central & $2,9 \%$ & $4,0 \%$ & $7,1 \%$ & $1,8 \%$ & $2,2 \%$ & $3,5 \%$ & $9,7 \%$ \\
\hline
\end{tabular}

Thus, this study provides, as proposed, the profile of cyclists in the city streets.

This population has $61 \%$ of its components with income between 1 and 5 minimum wages and on average 10 years of experience in the conduct of bicycles. It has an average age of 30 years and uses the bicycle as their primary means of transportation $(52.5 \%)$.

Despite the experience of cyclists, $20.5 \%$ of them have been involved in accidents, which repre- sent a rather high proportion, calling attention to the need for protective measures to those users. The accident type is diverse, drawing attention to the clash with small doors of automotive vehicles. Again, we highlight the conflict in the conflict in the use of shared use path, showing the need for the establishment of bike lanes.

Although the number of accidents involving cyclists, despite complaints about the lack of infrastructure and security, the rider of Juiz de Fora does not use individual safety equipment (67\%) nor the equipment mandated by the Brazilian Traffic Code - half of them do not have any of these items in your vehicle. However, more than $80 \%$ of the bicycles are equipped with multi-currency, reflecting the influence of local topography.

The topography of the city, though unfriendly, is not the main factor for the cyclist does not use your bike. The vast majority of respondents cited other factors as a hindrance to the traffic of bicycles in the city of Juiz de Fora.

Comparing the percentage of trips generated and attracted by the Administrative Regions, consolidates the position of the central area of the city as the main one. This feature shows that, when designing a network of bike paths in town, the main orientation should be neighborhood-center.

\section{References}

[1] Y. I TI, Percepção de risco dos ciclistas com relação ao sistema de tráfego urbano na cidade do Rio de Janeiro. Dissertação (Mestrado em Engenharia de Transportes), Universidade Federal do Rio de Janeiro, COPPE, Rio de Janeiro, 1997.

[2] P.A.C. Miguel, Metodologia da pesquisa em engenharia de produção e gestão de operações. Rio de Janeiro, Elsevier, 2010.

[3] D.C. Montgomery, G.C. Runger, Estatística aplicada e probabilidade para engenheiros, $2^{\mathrm{a}}$. Ed., Rio de Janeiro, LTC, 2003.

[4] E. Ferreira, Planejamento de Transporte Cicloviário: O caso de Cáceres - MT. Dissertação (Mestrado em Engenharia de Transportes), Universidade Federal do Rio de Janeiro, COPPE, Rio de Janeiro, 2005.

[5] A.C. Ferraz, I. Torres, Transporte Público Urbano. Editora Rima. São Carlos, 2004.

[6] P. Rietveld \& V. Daniel, "Determinants of bicycle use: do municipal policies matter?". Transportation Research, Part A 38. p. 531-550, 2004

[7] U. S Department of Transportation - U.S DOT. Guidebook on Methods to Estimate Non-Motorized Travel: Overview of Methods. 1999.

[8] Confederação Nacional da Indústria - CNI. Pesquisa CNIIBOPE: Retratos da Sociedade Brasileira: Locomoção Urbana. 2011. 